TRANSACTIONS OF THE

AMERICAN MATHEMATICAL SOCIETY

Volume 353, Number 5, Pages 2059-2072

S 0002-9947(01)02764-7

Article electronically published on January 10, 2001

\title{
IRREDUCIBLE PARTITIONS AND THE CONSTRUCTION OF QUASI-MEASURES
}

\author{
D. J. GRUBB
}

\begin{abstract}
A quasi-measure is a non-subadditive measure defined on only open or closed subsets of a compact Hausdorf space. We investigate the nature of irreducible partitions as defined by Aarnes and use the results to construct quasi-measures when $g(X)=1$. The cohomology ring is an important tool for this investigation.
\end{abstract}

\section{INTRODUCTION}

Let $X$ be a compact Hausdorff space and $C(X)$ the space of real-valued continuous functions on $X$. A function $\rho: C(X) \rightarrow \mathbf{R}$ is called quasi-linear if it is linear on each singly generated closed subalgebra of $C(X)$. In [1], Aarnes constructed the first example of a quasi-linear functional which is not linear. He did this by establishing a representation theorem of quasi-linear functionals in terms of "integrals" with respect to quasi-measures.

A quasi-measure on $X$ is a positive set function, $\mu$, defined on $\mathcal{A}$, the collection of sets which are either open or closed, such that:

a) $\mu(\emptyset)=0$,

b) If $A_{1} \subseteq A_{2}$, then $\mu\left(A_{1}\right) \leq \mu\left(A_{2}\right)$,

c) If $A=\bigcup_{i=1}^{n} A_{i}$ is a disjoint union, then $\mu(A)=\sum_{i=1}^{n} \mu\left(A_{i}\right)$,

d) If $U$ is open, then $\mu(U)=\sup \{\mu(C): C \subseteq U, C$ closed $\}$.

It is important to emphasize that the third condition only applies when $A \in \mathcal{A}$. In general, it is impossible to consistently define a quasi-measure on sets outside of $\mathcal{A}$. In fact, a quasi-measure extends to a measure on a sigma-algebra only if sets of the form $C \cap U$ can be consistently measured where $C$ is closed and $U$ is open.

In [2], Aarnes obtained a general construction procedure for quasi-measures which we now outline. We will assume from now on that $X$ is both connected and locally connected as well as being compact. Our ultimate goal is to use Aarnes' procedure to construct new quasi-measures.

Definition 1. A subset $A \subseteq X$ is said to be solid if both $A$ and $X \backslash A$ are connected. An irreducible partition $\mathcal{P}=\left\{C_{i}\right\}_{1}^{n} \cup\left\{U_{\alpha}\right\}_{\alpha \in A}$ is a collection of disjoint solid sets such that:

(1) Each $C_{i}$ is closed and each $U_{\alpha}$ is open,

Received by the editors March 3, 1998 and, in revised form, April 25, 2000.

2000 Mathematics Subject Classification. Primary 28C15, 55N45, 46G12.

Key words and phrases. Quasi-measure, irreducible partition, cup product.

(C)2001 American Mathematical Society 
(2) We have $X=\bigcup_{1}^{n} C_{i} \cup \bigcup_{\alpha \in A} U_{\alpha}$,

(3) If $J \subsetneq\{1, \cdots, n\}$, then $X \backslash \bigcup_{i \in J} C_{i}$ is connected.

Notice that there are only finitely many closed solid sets, but potentially infinitely many open solid sets in an irreducible partition. We will see later that there are, in fact, only finitely many open sets. If there is only one closed set, i.e. $n=1$, we say the partition $\mathcal{P}$ is trivial. In this case $\mathcal{P}=\{C, X \backslash C\}$ for some closed solid set C.

We define $g(X)$ to be the maximum value of $n-1$ for irreducible partitions $\mathcal{P}=\left\{C_{i}\right\}_{1}^{n} \cup\left\{U_{\alpha}\right\}_{\alpha \in A}$ of $X$. Similarly, we let $o(X)$ be the maximum value of $\operatorname{card}(A)-1$ for such partitions. In particular, $g(X)=0$ if and only if every partition of $X$ is trivial, in which case $o(X)=0$ also.

We will need two results from [2].

Proposition 2. If $C \subseteq X$ is solid and $C \subseteq U$ is open, then there is an open solid set $W$ and a closed solid set $K$ with $C \subseteq W \subseteq K \subseteq U$.

Proposition 3. If $\left\{C_{i}\right\}_{1}^{n}$ is a collection of closed solid sets such that $X \backslash \bigcup_{1}^{n} C_{i}$ is disconnected, but for every $J \subsetneq\{1, \cdots, n\}$ a proper subset, $X \backslash \bigcup_{i \in J} C_{i}$ is connected, then the components $\left\{U_{\alpha}\right\}$ of $X \backslash \bigcup_{1}^{n} C_{i}$ are solid and so $\left\{C_{i}\right\}_{1}^{n} \cup\left\{U_{\alpha}\right\}_{\alpha \in A}$ is an irreducible partition of $X$. We will say that $\left\{C_{i}\right\}_{1}^{n}$ generates an irreducible partition.

The construction in [2] proceeds as follows. Let $\mathcal{A}_{s}$ denote the collection of solid sets which are either open or closed. We say $\mu: \mathcal{A}_{s} \rightarrow[0,1]$ is a solid set function if

(1) If $C_{1}, C_{2}, \cdots, C_{n}$ are disjoint, closed, solid sets contained in the closed, solid set $C$, then $\sum_{1}^{n} \mu\left(C_{i}\right) \leq \mu(C)$,

(2) If $U$ is solid and open, then $\mu(U)=\sup \{\mu(C): C \subseteq U, C$ closed, solid $\}$,

(3) If $\left\{C_{i}\right\}_{1}^{n} \cup\left\{U_{\alpha}\right\}_{\alpha \in A}$ is an irreducible partition of $X$, then

$$
\sum_{1}^{n} \mu\left(C_{i}\right)+\sum_{\alpha \in A} \mu\left(U_{\alpha}\right)=\mu(X)=1 .
$$

The main result of [2] is the following theorem.

Theorem 4. Every solid set function extends uniquely to a quasi-measure on $X$.

Typically, the first two conditions in the definition of a solid set function are straightforward to check, but the third causes difficulties because the irreducible partitions of $X$ must be known. For this reason, most known examples of quasimeasures occur in spaces such as the sphere or unit square where $g(X)=0$. However, Knudsen managed to find a quasi-measure on the torus, where $g(X)=1$. See 4] for details.

Our main goal, achieved in the last section of this paper is to give an explicit construction of solid set functions on a variety of spaces with $g(X)=1$. While this may seem like a modest task, the examples of this paper are the only quasimeasures known for spaces with $g(X)=1$ except for those that are images from spaces with $g(X)=0$. The amount of work expended to produce these examples seems, at this time, to be necessary. 


\section{IRREDUCIBLE PARTITIONS}

In this section we present some basic properties of irreducible partitions. Our primary goal is to find a simpler way of determining $g(X)$ for a given space $X$. In particular, we show that $g(X)$ is related to the "sidedness" of solid sets.

Proposition 5. If $\mathcal{P}=\left\{C_{i}\right\}_{1}^{n} \cup\left\{U_{\alpha}\right\}_{\alpha \in A}$ is an irreducible partition of $X$, then $C_{i} \cap \bar{U}_{\alpha} \neq \emptyset$ for all $1 \leq i \leq n$ and $\alpha \in A$.

Proof. Suppose that $C_{i}$ and $\bar{U}_{\alpha}$ are disjoint. Then the boundary of $U_{\alpha}$ is contained in $\bigcup_{j \neq i} C_{j}$, so $U_{\alpha} \cup \bigcup_{j \neq i} C_{j}=\bar{U}_{\alpha} \cup \bigcup_{j \neq i} C_{j}$ is closed.

This shows that $X \backslash \bigcup_{j \neq i} C_{j}=U_{\alpha} \cup\left[X \backslash\left(U_{\alpha} \cup \bigcup_{j \neq i} C_{j}\right)\right]$ is a disjoint union of open sets. Since $U_{\alpha} \neq \emptyset$ and $C_{i}$ is disjoint from $U_{\alpha} \cup \bigcup_{j \neq i} C_{j}$, we see that $X \backslash \bigcup_{j \neq i} C_{j}$ is disconnected. This contradicts that $\mathcal{P}$ is an irreducible partition.

Corollary 6. If $\left\{C_{i}\right\}_{1}^{n} \cup\left\{U_{\alpha}\right\}_{\alpha \in A}$ is an irreducible partition of $X$ and $B \subsetneq A$ is a proper subset, then $X \backslash \bigcup_{\alpha \in B} U_{\alpha}$ is connected.

Proof. We have $X \backslash \bigcup_{\alpha \in B} U_{\alpha}=\bigcup_{1}^{n} C_{i} \cup \bigcup_{\alpha \notin B} U_{\alpha}$ is connected by the proposition since each $C_{i}$ and $U_{\alpha}$ is connected.

Proposition 7. Let $\left\{C_{i}\right\}_{1}^{n} \cup\left\{U_{\alpha}\right\}_{\alpha \in A}$ be an irreducible partition of $X$. Then card $A$ (the number of open sets) is finite.

Proof. First pick disjoint open sets $W_{i}$ with $C_{i} \subseteq W_{i}$. Then, by the previous proposition, $W_{i} \cap U_{\alpha} \neq \emptyset$ for each $1 \leq i \leq n$ and $\alpha \in A$. Since $U_{\alpha}$ is connected, we cannot have $U_{\alpha} \subseteq \bigcup_{i=1}^{n} W_{i}$ for any $\alpha$. If we pick $x_{\alpha} \in U_{\alpha} \backslash\left(\bigcup_{i=1}^{n} W_{i}\right)$, then $\left\{x_{\alpha}\right\}_{\alpha \in A}$ cannot have a limit point in any $U_{\alpha}$, nor in any $W_{i}$. Since these sets cover $X$, we have a contradiction if $A$ is infinite.

Thus there is a certain amount of symmetry between the open sets and the closed sets in an irreducible partition. We will see more examples of this as we proceed. Now we give a fundamental definition for our investigation.

Definition 8. Let $C$ be a closed solid set in $X$. We say that $C$ has at least $n$ sides if there is an open set $C \subseteq U$ such that whenever $W$ is open with $C \subseteq W \subseteq U$, then $W \backslash C$ has at least $n$ connected components. If $C$ has exactly $n$ sides, we write $\operatorname{sd}(C)=n$. In this case, there is an open set $U$ containing $C$ such that whenever $W$ is a connected open subset with $C \subseteq W \subseteq U, W \backslash C$ has exactly $n$ connected components. We let $s(X)$ denote the largest number of sides of any closed, solid set in $X$. We allow $s(X)$ to be infinite, but we do not distinguish between different infinite cardinalities. Finally, if $U$ is an open solid set, we define the number of sides of $U$ to be the same as the number of sides of $X \backslash U$.

As an example, the set $[0,1] \times\{x\}$ is two-sided in the annulus $[0,1] \times S^{1}$ for every $x \in S^{1}$. On the other hand, the sets $\{0\} \times S^{1}$ and $\{1\} \times S^{1}$ are one-sided in the annulus. There is a 3 -sided solid set in the two-holed annulus.

There are spaces where $s(X)=+\infty$ yet every closed, solid set has only finitely many sides. Such a space can be obtained by considering the join of $n$-holed annuli, one for each $n$.

Proposition 9. Let $\left\{C_{i}\right\}_{1}^{n} \cup\left\{U_{j}\right\}_{1}^{m}$ be an irreducible partition of $X$. Then $\operatorname{sd}\left(C_{i}\right) \geq$ $m$ for each $i$. 
Proof. Let $U=X \backslash \bigcup_{j \neq i} C_{j}$. Then, if $C_{i} \subseteq W \subseteq U$, we have $W \backslash C_{i}=\bigcup_{1}^{m} W \cap U_{j}$. By proposition 5 each $W \cap U_{j}$ is non-empty. Since the terms in the union are disjoint, $W \backslash C_{i}$ has at least $m$ components.

Corollary 10. A one-sided closed set can only be in trivial irreducible partitions.

There is a type of regularity with respect to closed sets with at least $n$ sides as described in the following proposition.

Proposition 11. Let $C$ be a closed, solid set with at least $n \geq 2$ sides. Suppose that $K$ is a closed set with $C \cap K=\emptyset$. Then there is a closed, solid set $C^{\prime}$ containing $K$ and disjoint from $C$ with $\operatorname{sd}\left(C^{\prime}\right) \geq n$. In fact, $C^{\prime}$ may be chosen so that $\left\{C, C^{\prime}\right\}$ generates an irreducible partition with at least $n$ open sets.

Proof. Use proposition 2 to obtain an open, solid set $W$ with $C \subseteq W$ and $W \cap K=\emptyset$ such that $W \backslash C$ has at least $n$ connected components. Let $C^{\prime}=X \backslash W$. Then $X \backslash\left(C \cup C^{\prime}\right)=W \backslash C$ is disconnected so by proposition 3, $\left\{C, C^{\prime}\right\}$ generates an irreducible partition with at least $n$ open sets. Hence $C^{\prime}$ has at least $n$ sides and $K \subseteq C^{\prime}$.

Corollary 12. We have $o(X)=s(X)-1$.

Proof. This follows immediately from the last two propositions.

The next proposition is analogous to a previous result for closed sets.

Proposition 13. If $\left\{C_{i}\right\}_{1}^{n} \cup\left\{U_{j}\right\}_{1}^{m}$ is an irreducible partition of $X$, then $\operatorname{sd}\left(U_{j}\right) \geq$ $n$ for each $j$.

Proof. Pick disjoint open, solid sets $W_{i}$ with $C_{i} \subseteq W_{i}$ for $1 \leq i \leq n$. Let $U=$ $\bigcup_{k \neq j} U_{k} \cup \bigcup_{1}^{n} W_{i}$. Then $U$ is an open set containing $X \backslash U_{\alpha}$. If $V$ is open with $X \backslash U_{j} \subseteq V \subseteq U$, then each $V \cap W_{i}$ is an open set containing $C_{i}$, so $V \cap W_{i} \cap U_{j}$ is non-empty by proposition 5. Thus, $V \backslash\left(X \backslash U_{j}\right)=V \cap U_{j}=\bigcup_{1}^{n} V \cap W_{i} \cap U_{j}$ has at least $n$ components.

We can now construct a solid set function on fairly general spaces. Let $F \subseteq X$ be a closed subset such that $F \cap C \neq \emptyset$ whenever $\operatorname{sd}(C) \geq 2$. For example, if $X=S^{1} \times[0,1]$, we may choose $F=S^{1} \times\{0\}$. Let $p \notin F$ be any point of $X$. We may define a set function on solid sets $\mu$ by $\mu(A)=1$ if $F \subseteq A$ or if $p \in A$ and $A \cap F \neq \emptyset$. Define $\mu(A)=0$ otherwise. It is easily seen that $\mu$ then defines a solid set function and hence extends to a quasi-measure on $X$. Notice that if $g(X)=0$, the condition on $F$ trivializes. The first example of a non-linear quasi-linear functional was obtained from a quasi-measure of this type. See [1] for details. It is crucial to point out that for a general closed set, it is possible for $\mu(C)=1$ even if $p \notin C$ and $C \cap F=\emptyset$. The above definition only applies to solid sets.

Theorem 14. Suppose that $\left\{C_{i}\right\}_{1}^{n} \cup\left\{U_{j}\right\}_{1}^{m}$ is an irreducible partition of $X$. Then there exists another irreducible partition $\left\{D_{j}\right\}_{1}^{m} \cup\left\{V_{i}\right\}_{1}^{n}$ such that $D_{j} \subseteq U_{j}$ for each $j \leq m$ and $C_{i} \subseteq V_{i}$ for each $i \leq n$. Furthermore, if each set of the original partition has finitely many sides, the new partition may be chosen so that each $U_{j} \backslash D_{j}$ has $\operatorname{sd}\left(U_{j}\right)$ components and each $V_{i} \backslash C_{i}$ has $\operatorname{sd}\left(C_{i}\right)$ components.

Proof. Pick disjoint open, solid sets $\left\{W_{i}\right\}_{1}^{n}$ with $C_{i} \subseteq W_{i}$ for each $i$. If $C_{i}$ has finitely many sides, choose $W_{i}$ so that $W_{i} \backslash C_{i}$ has $\operatorname{sd}\left(C_{i}\right)$ components. By proposition 5 
each $W_{i} \cap U_{j} \neq \emptyset$. Since each $U_{j}$ is connected, we have that $U_{j} \backslash\left(\bigcup_{1}^{n} W_{i}\right)$ is nonempty. By proposition 2 we may find a closed, solid set $D_{j}$ with $U_{j} \backslash\left(\bigcup_{1}^{n} W_{i}\right) \subseteq$ $D_{j} \subseteq U_{j}$. If $U_{j}$ has finitely many sides, we may choose $D_{j}$ so that $U_{j} \backslash D_{j}$ has $\operatorname{sd}\left(U_{j}\right)$ components. In any case, we can obtain that $U_{j} \backslash D_{j}$ is disconnected. We will show that $\left\{D_{j}\right\}_{1}^{m}$ generates an irreducible partition with the required properties.

First notice that $\bigcup_{1}^{n} C_{i} \subseteq X \backslash \bigcup_{1}^{m} D_{j} \subseteq \bigcup_{1}^{n} W_{i}$, so $X \backslash \bigcup_{1}^{m} D_{j}$ is disconnected (recall that $C_{i} \subseteq W_{i}$ ). In fact, it has at least $n$ components.

Next, if $J \subsetneq\{1, \cdots, m\}$ is a proper subset, then $X \backslash \bigcup_{j \in J} U_{j}$ is connected by corollary 6. We have $X \backslash \bigcup_{j \in J} D_{j}=\left(X \backslash \bigcup_{j \in J} U_{j}\right) \cup \bigcup_{j \in J}\left(U_{j} \backslash D_{j}\right)$.

Since, $U_{j} \backslash D_{j}$ is disconnected, $\left\{D_{j}, X \backslash U_{j}\right\}$ generates an irreducible partition of $X$. Let $U_{j} \backslash D_{j}=\bigcup_{k=1}^{m_{j}} V_{j k}$ be the decomposition into connected components where each $V_{j k}$ is solid. Then, again by proposition 5 , we have $\emptyset \neq \bar{V}_{j k} \cap X \backslash U_{j} \subseteq$ $\bar{U}_{j} \backslash U_{j} \subseteq X \backslash \bigcup_{j \in J} U_{j}$. Thus $\bar{V}_{j k} \cap X \backslash \bigcup_{j \in J} U_{j}$ is non-empty. Since both $V_{j k}$ and $X \backslash \bigcup_{j \in J} U_{j}$ are connected, so is $\left(X \backslash \bigcup_{j \in J} U_{j}\right) \cup V_{j k}$.

We next have that $X \backslash \bigcup_{j \in J} D_{j}=\bigcup_{j, k}\left[\left(X \backslash \bigcup_{j \in J} U_{j}\right) \cup V_{j k}\right]$ is connected. By proposition 3 we see that $\left\{D_{j}\right\}_{1}^{m}$ generates an irreducible partition of $X$. Since $X \backslash \bigcup_{1}^{m} D_{j}$ has at least $n$ components, there are at least $n$ open solid sets in this partition. We may list these components so that $C_{i} \subseteq V_{i} \subseteq W_{i}$ for $i \leq n$. Then, by choice of $W_{i}, V_{i} \backslash C_{i}$ has $\operatorname{sd}\left(C_{i}\right)$ components.

The only remaining issue is whether there are more than $n$ components to $X \backslash \bigcup_{1}^{m} D_{j}$. If $V$ is a component different from each $V_{i}$ with $i \leq n$, then $V$ is also disjoint from each $C_{i}$. But this shows that $V \subseteq \bigcup_{1}^{m} U_{j} \backslash D_{j}$. Hence $V \subseteq V_{j k}$ for some $j, k$. But then $\bar{V} \cup D_{j_{0}}=\emptyset$ for $j_{0} \neq j$. This contradicts Proposition 5 .

Corollary 15. We have $g(X)=o(X)=s(X)-1$.

Corollary 16. The quantity $g(X)+1$ is equal to the maximum number of components of a set of the form $V \cap W$ where $V$ and $W$ are open solid sets with $V \cup W=X$.

Proof. This is easily seen from proposition 11 by letting $C=X \backslash V$ and $C^{\prime}=$ $X \backslash W$.

As we shall see, this characterization allows the use of algebraic topological techniques to evaluate $g(X)$.

Corollary 17. Let $\mathcal{P}=\left\{C_{i}\right\}_{1}^{n} \cup\left\{U_{j}\right\}_{1}^{m}$ be an irreducible partition of $X$ where all sets are finitely sided. Then

$$
\sum_{1}^{n} \operatorname{sd}\left(C_{i}\right)=\sum_{1}^{m} \operatorname{sd}\left(U_{j}\right)
$$

We call the common value the trace of the partition and denote it by $\operatorname{tr}(\mathcal{P})$.

Proof. This follows immediately from the theorem by noticing that

$$
\bigcup_{1}^{n}\left(V_{i} \backslash C_{i}\right)=\bigcup_{1}^{m}\left(U_{j} \backslash D_{j}\right)
$$




\section{Cohomology AND IRREduciBle partitions}

It is worthwhile at this point to consider what is involved when determining the irreducible partitions of a space $X$, or even the value of $g(X)$. For example, if $X=[0,1]^{2}$, then $g(X)=0$, although even this simple fact is not obvious and requires some algebraic topology. In [4], it was shown that for CW-complexes, $g(X)=0$ if and only if the cohomology module $H^{1}(X)=0$. This is what justifies the heavy reliance on the cohomology ring in this paper.

In this section, we will assume that $X$ is locally path connected in addition to being connected and compact. We will be primarily interested in the cohomology modules $\tilde{H}^{0}(V)$ and $H^{1}(V)$ for $V$ open in $X$. It is also assumed that all coefficient rings are principal ideal domains. We start with a definition.

Definition 18. A submodule $L \subseteq H^{1}(X)$ will be called isotropic if whenever $\alpha, \beta \in$ $L$, we have $\alpha \cup \beta=0$ in $H^{2}(X)$. Here, $\alpha \cup \beta$ is the cohomology cup product of $\alpha$ and $\beta$. We let $d(X)$ be the maximum rank of an isotropic submodule of $H^{1}(X)$.

Notice that if $H^{2}(X)=0$, we have $d(X)=\operatorname{rank} H^{1}(X)$. In any case $d(X) \leq$ $\operatorname{rank} H^{1}(X)$.

We need one crucial, but elementary, fact about cup products. If $V$ and $W$ are open sets of $X$ with $V \cup W=X$, and if $i_{V}, i_{W}$ are the maps on cohomology induced by the inclusions, then $\alpha \cup \beta=0$ whenever $\alpha \in \operatorname{ker} i_{V}$ and $\beta \in \operatorname{ker} i_{W}$. This follows easily from the naturality properties of the cup product on pairs of spaces. In particular, then $\operatorname{ker} i_{V} \cap \operatorname{ker} i_{W}$ is an isotropic submodule.

Theorem 19. We have $s(X) \leq 1+d(X)$.

Proof. Let $C$ be any closed solid set and let $W$ be an open solid set containing $C$. Let $V=X \backslash C$. Then $V \cup W=X$, so the Mayer-Vietoris sequence is exact:

$$
\rightarrow \tilde{H}^{0}(V) \oplus \tilde{H}^{0}(W) \rightarrow \tilde{H}^{0}(V \cap W) \rightarrow H^{1}(X) \rightarrow H^{1}(V) \oplus H^{1}(W) \rightarrow .
$$

Since $V$ and $W$ are solid, they are connected, so $\tilde{H}^{0}(V)=\tilde{H}^{0}(W)=0$. Thus the boundary map from $\tilde{H}^{0}(V \cap W)$ to $H^{1}(X)$ is injective. By exactness, the image is the kernel $L=\operatorname{ker} i_{V} \cap \operatorname{ker} i_{W}$. But $L$ is an isotropic submodule. Since the rank of $\tilde{H}^{0}(V \cap W)$ is one less than the number of components of $V \cap W$, we see that the number of components of $W \backslash C=V \cap W$ is less than $1+\operatorname{rank} L \leq 1+d(X)$.

Corollary 20. We always have $g(X) \leq d(X)$.

Using corollary [16 and a calculation as in the proof of the theorem, we see that $g(X)$ is the maximum value of $\operatorname{rank}\left(\operatorname{ker} i_{V} \cap \operatorname{ker} i_{W}\right)$ where $V$ and $W$ range over the open solid sets $V$ and $W$ where $V \cup W=X$.

Proposition 21. If $X$ and $Y$ are spaces with $H^{*}(X)$ and $H^{*}(Y)$ of finite type and the coefficient ring is a field, then $d(X \times Y)=\max \{d(X), d(Y)\}$.

Proof. In this situation, we may apply the Künneth formula to see that an element of $H^{1}(X \times Y)$ is of the form $\alpha \times 1+1 \times \beta$, where $\alpha \in H^{1}(X)$ and $\beta \in H^{1}(Y)$. The cup product is given by the formula

$$
\begin{aligned}
& \left(\alpha_{1} \times 1+1 \times \beta_{1}\right) \cup\left(\alpha_{2} \times 1+1 \times \beta_{2}\right) \\
& \quad=\left(\alpha_{1} \cup \alpha_{2}\right) \times 1+\alpha_{1} \times \beta_{2}-\alpha_{2} \times \beta_{1}+1 \times\left(\beta_{1} \cup \beta_{2}\right) .
\end{aligned}
$$

By the independence parts of the Künneth formula, this can be 0 only when $\alpha_{1} \cup$ $\alpha_{2}=0, \beta_{1} \cup \beta_{2}=0$, and $\alpha_{1} \times \beta_{2}=\alpha_{2} \times \beta_{1}$. But this requires either that $\alpha_{1} \times 1+1 \times \beta_{1}$ 
is a scalar multiple of $\alpha_{2} \times 1+1 \times \beta_{2}$, or that $\beta_{1}=\beta_{2}=0$, or $\alpha_{1}=\alpha_{2}=0$. This gives the required result.

Examples. a) If $H^{1}(X)=0$, we see that $g(X)=0$. This, and the converse for CW-complexes, was proved by Knudsen in [4].

b) If $X$ is a compact, 2-dimensional manifold without boundary, we take coefficients in the ring $\mathbf{Z} /(2)$. Since $H^{2}(X)=\mathbf{Z} /(2)$, the cup product on $H^{1}(X)$ induces an alternating bilinear form. It is a well-known consequence of Poincaré duality that this form is non-degenerate. A subspace is isotropic under our definition if and only if it is isotropic under this bilinear form, see [3]. This justifies our terminology. In this setting, $d(X)$ is called the Witt index of $H^{1}(X)$. It is known that this index is less than half the dimension of $H^{1}(X)$ over $\mathbf{Z} /(2)$. In particular, if $X$ is the oriented 2-manifold of genus $n$, then $g(X) \leq n$. If $X$ is the non-oriented manifold obtained by attaching $n$ crosscaps to the sphere, then $g(X) \leq[n / 2]$. Examples of closed solid sets with the required number of sides show both of these are equalities.

c) If $X$ is the $n$-holed annulus, $\operatorname{rank} H^{1}(X)=n$, so $g(X) \leq n$. Here, it is easy to construct an irreducible partition of $X$ with $n$ closed sets.

d) Our definition of $d(X)$ does depend on the coefficient ring. In fact, if $X$ is the non-orientable surface obtained by attaching $n$ cross caps to a sphere, then with coefficients in the rationals, $\mathbf{Q}, d(X)=n-1$. However, with any field as coefficient ring, $d(X)=g(X)=1$ when $X$ is the circle, the Möbius strip, or the Klein bottle.

e) It is possible for $d(X) \neq g(X)$ for every coefficient ring. For example, if $X$ is the space consisting of two tangent circles, $d(X)=2$, while $g(X)=1$.

Proposition 22. Let $\left\{C_{i}\right\}_{1}^{n}$ be a collection of disjoint, closed, solid sets such that $X \backslash \bigcup_{1}^{n} C_{i}$ is connected. Let $s_{i}$ be the number of sides of $C_{i}$. Then

$$
\sum_{1}^{n} s_{i} \leq n+d(X)
$$

This provides another way to see that $g(X) \leq d(X)$, since if $\left\{C_{i}\right\}_{1}^{n} \cup\left\{U_{j}\right\}_{1}^{m}$ is a non-trivial irreducible partition of $X$, each $s_{i} \geq 2$ and $\left\{C_{i}\right\}_{1}^{n-1}$ satisfies the conditions of the proposition.

Proof. Let $C_{i} \subseteq W_{i}$ be disjoint open, solid sets. Let $W=\bigcup_{1}^{n} W_{i}$ and $V=X \backslash \bigcup_{1}^{n} C_{i}$. Then $V$ is connected and $W$ has $n$ components. Also, $W \cup V=X$ and $W \cap V=$ $\bigcup_{1}^{n}\left(W_{i} \backslash C_{i}\right)$. Using the Mayer-Vietoris sequence, we obtain the exact sequence

$$
0 \rightarrow H^{0}(X) \rightarrow H^{0}(V) \oplus H^{0}(W) \rightarrow H^{0}(V \cap W) \rightarrow \operatorname{ker} i_{V} \cap \operatorname{ker} i_{W} \rightarrow 0 .
$$

Thus we have, by taking the alternating sum of the ranks, that

$$
1-(1+n)+\operatorname{rank} H^{0}(V \cap W)-\operatorname{rank}\left(\operatorname{ker} i_{V} \cap \operatorname{ker} i_{W}\right)=0 .
$$

Since $\operatorname{ker} i_{V} \cap \operatorname{ker} i_{W}$ is an isotropic submodule, we have

$$
\operatorname{rank} H^{0}(V \cap W)=n+\operatorname{rank}\left(\operatorname{ker} i_{V} \cap \operatorname{ker} i_{W}\right) \leq n+d(X) .
$$

If we now let $W_{i}$ shrink down to $C_{i}$, the left hand side is $\sum_{1}^{n} s_{i}$.

Another application of the Mayer-Vietoris sequence provides the following proposition. 
Proposition 23. Let $\mathcal{P}=\left\{C_{i}\right\}_{1}^{n} \cup\left\{U_{j}\right\}_{1}^{m}$ be an irreducible partition of $X$. Then

$$
\operatorname{tr}(\mathcal{P}) \leq d(X)+n+m-1 \text {. }
$$

Proof. Let $\left\{D_{j}\right\}_{1}^{m} \cup\left\{V_{i}\right\}_{1}^{n}$ be a partiton as in theorem 14. Set $V=\bigcup_{1}^{n} V_{i}$ and $W=\bigcup_{1}^{m} U_{j}$. Then $V \cap W=\bigcup_{1}^{m}\left(U_{j} \backslash D_{j}\right)$ has $\operatorname{tr}(\mathcal{P})$ components. The result follows as in the previous proof.

Proposition 24. Let $\left\{C_{i}\right\}_{1}^{n} \cup\left\{U_{j}\right\}_{1}^{m}$ be an irreducible partition of $X$ with $n \geq 3$. If $1 \leq i \neq j \leq n$ and let $B=\bigcup_{1}^{m} U_{j} \cup C_{i} \cup C_{j}$, then

$$
\operatorname{rank} H^{1}(B) \geq m-1 \text {. }
$$

Proof. Let $V=X \backslash \bigcup_{k \neq i} C_{k}$ and $W=X \backslash \bigcup_{k \neq j} C_{k}$. Then $V \cap W=\bigcup_{1}^{m} U_{j}$ and $V \cup W=B$. Also, both $V$ and $W$ are connected, so

$$
0=\tilde{H}^{0}(V) \oplus \tilde{H}^{0}(W) \rightarrow \tilde{H}^{0}\left(\bigcup_{1}^{m} U_{j}\right) \rightarrow H^{1}(B) \rightarrow
$$

gives the result.

We define a space $X$ to be a Witt space for a coefficient ring if $g(X)=d(X)$. It seems natural to ask which spaces are Witt spaces and which are not. The classification theorem and explicit examples of solid sets shows that all 2-manifolds with boundary are Witt spaces. We notice that $A \times Y$ is solid in $X \times Y$ if $Y$ is connected and $A$ is solid in $X$. Hence, we always have that $g(X \times Y) \geq \max \{g(X), g(Y)\}$, the product of two Witt spaces is again a Witt space as long as the Künneth Theorem holds. In particular, any space of the form $X=\left(S^{1}\right)^{n}$ has $d(X)=g(X)=1$.

In the case of Witt spaces, the last few results put very stringent conditions on the sets in an irreducible partition. For example, if $X$ is a Witt space with $g(X)=1$, then any two disjoint, closed, two-sided sets disconnect the space by proposition 22 and hence generate an irreducible partition. There can only be two open sets in this partition, each of which is 2-sided by corollary 17

On the other hand, if $X$ is a Witt space with $g(X)=2$, then two 2-sided closed sets need not disconnect, but three automatically do. In the latter case, each open set must have at least three sides, so by using the trace, there must be two such open sets. Continuing in this manner, it is possible to classify all the possible irreducible partitions for spaces with small values of $g(X)$. This, however, does not easily yield quasi-measures since the containment properties of the sets must also be considered.

We next turn to a more detailed analysis of how closed solid sets are positioned in the overall space.

If $C$ is any closed set, we may construct the Alexander cohomology of $C$ by the direct limit

$$
\check{H}^{k}(C)=\lim _{V} H^{k}(V)
$$

where $V$ ranges over the open sets containing $C$. If $C$ is solid, the open solid sets are cofinal in the collection of all open sets containing $C$, so we may obtain $\check{H}^{k}(C)$ by taking the direct limit over only open solid sets. Another interesting cohomology module associated with $C$ is the direct limit

$$
\check{H}_{s}^{k}(C)=\lim _{V} H^{k}(V \backslash C) .
$$


We call this module the cohomology of the sides of $C$. This cohomology module is sensitive to the area near $C$. In fact, $\check{H}_{s}^{0}(C)$ is free with as many generators as $C$ has sides. Both of the cohomology modules above have analogous reduced cohomology modules.

Proposition 25. Let $C \subseteq X$ be solid. There is an exact sequence

$$
\rightarrow H^{k}(X) \rightarrow \check{H}^{k}(C) \oplus H^{k}(X \backslash C) \rightarrow \check{H}_{s}^{k}(C) \rightarrow H^{k+1}(X) \rightarrow
$$

which can be initiated with reduced cohomology modules.

Proof. Let $V=X \backslash C$. If $C \subseteq W$, we have a Mayer-Vietoris sequence

$$
\rightarrow H^{k}(X) \rightarrow H^{k}(W) \oplus H^{k}(V) \rightarrow H^{k}(W \cap V) \rightarrow H^{k+1}(X) \rightarrow .
$$

Since $W \cap V=W \backslash C$, we obtain the result by taking direct limits.

In particular, if $i_{C}: H^{1}(X) \rightarrow \check{H}^{1}(C)$ is the map induced by inclusion, and $V=X \backslash C$, we see that the rank of $L=\operatorname{ker} i_{C} \cap \operatorname{ker} i_{V}$ is one less than the number of sides of $C$. Furthermore, $L$ is an isotropic submodule of $H^{1}(X)$. In fact, if $\alpha \in \operatorname{ker} i_{C}$ and $\beta \in \operatorname{ker} i_{V}$, there is a solid set $W$ containing $C$ with $i_{W}(\alpha)=0$. Since $V \cup W=X, \alpha \cup \beta=0$. We call $L$ the isotropic submodule associated with $C$.

Theorem 26. Let $X$ be a Witt space with $g(X)=1$ and let $C_{1}$ and $C_{2}$ be two disjoint closed solid, two-sided sets. If $L_{1}$ and $L_{2}$ are the isotropic submodule associated with $C_{1}$ and $C_{2}$, then $L_{1}+L_{2}$ has rank one. In particular, if the coefficient ring is a field, $L_{1}=L_{2}$. Moreover, if the coefficient ring is a field of characteristic not equal to 2 , then $L_{1}=\operatorname{ker} i_{C_{1}}=\operatorname{ker} i_{X \backslash C_{1}}$. Similarly for $L_{2}$.

Proof. Let $V_{i}=X \backslash C_{i}$. Since $C_{1}$ is two-sided, $L_{1}=\operatorname{ker} i_{C_{1}} \cap \operatorname{ker} i_{V_{1}}$ has rank one by the comments after proposition 25 Let $\alpha \in L_{1}$ and $\beta \in L_{2}$. Since $V_{1} \cup V_{2}=X$, $\alpha \in \operatorname{ker} i_{V_{1}}$ and $\beta \in \operatorname{ker} i_{V_{2}}$, we must have that $\alpha \cup \beta=0$. We also have that $\alpha \cup \alpha=\beta \cup \beta=0$ since $L_{1}$ and $L_{2}$ are isotropic. This shows that $L_{1}+L_{2}$ is an isotropic submodule of $H^{1}(X)$. Thus $L_{1}+L_{2}$ has rank one.

Now assume that the coefficient ring is a field of characteristic not 2 and let $\alpha \in \operatorname{ker} i_{V_{1}}$ and $\beta \in \operatorname{ker} i_{V_{2}}$. As before, $\alpha \cup \beta=0$. But now $\alpha \cup \alpha=\beta \cup \beta=0$ because the field is not of characteristic two. Thus, the space spanned by $\alpha$ and $\beta$ is isotropic. Since $d(X)=1$, we must have that $\alpha$ is a scalar multiple of $\beta$. Since this happens for all $\alpha$ and $\beta$, we must have that $\operatorname{ker} i_{V_{1}}=\operatorname{ker} i_{V_{2}}$. But this means that both of these subspaces are one dimensional since ker $i_{V_{1}} \cap \operatorname{ker} i_{V_{2}}$ is isotropic.

But now, $L_{1}=\operatorname{ker} i_{C_{1}} \cap \operatorname{ker} i_{V_{1}}$ is one dimensional. Since $\operatorname{ker} i_{V_{1}}$ is also one dimensional, $\operatorname{ker} i_{V_{1}} \subseteq \operatorname{ker} i_{C_{1}}$.

Now suppose that $\alpha \in \operatorname{ker} i_{C_{1}}$. Then there is an open solid set $W$ containing $C_{1}$ such that $\alpha \in \operatorname{ker} i_{W}$. By definition of two sidedness, we may assume that $W \backslash C_{1}$ has two components. Thus $\left\{C_{1}, X \backslash W\right\}$ generates an irreducible partition of $X$. By the above, we have that $\operatorname{ker} i_{V_{1}}=\operatorname{ker} i_{W}$ contains $\alpha$. Thus $\operatorname{ker} i_{C_{1}} \subseteq \operatorname{ker} i_{V_{1}} \subseteq \operatorname{ker} i_{C_{1}}$. This completes the proof.

Thus, we may classify the closed solid sets in $X$ by considering the associated isotropic subspaces. We also see that every set in an irreducible partition has the same associated subspace. This will ultimately allow the construction of some interesting quasi-measures. The basic idea is to have solid sets with different associated isotropic subspaces be measured by different measures. The details are the substance of the next section. 


\section{QUASI-MEASURES WHEN $g(X)=1$}

In this section we will asssume that $X$ is a compact, connected, locally connected Hausdorff space with $g(X)=1$. We will also assume that whenever $C$ and $C^{\prime}$ are disjoint closed, solid, two-sided sets, then $\left\{C, C^{\prime}\right\}$ generates an irreducible partition of $X$.

This last condition is non-trivial. For example, if $X$ consists of two tangent circles, we have that $g(X)=1$, but closed solid sets in different circles will not disconnect the space. However, if $X$ is any locally path connected Witt space with $g(X)=1$, our hypothesis is satisfied by proposition 22 .

We let $\mathcal{C}_{s}$ denote the collection of all closed solid sets in $X$. Similarly, we let $\mathcal{C}_{s 1}$ (resp. $\mathcal{C}_{s 2}$ ) denote the collection of all one-sided (resp. two-sided) closed solid sets. Since $s(X)=1+g(X)=2, \mathcal{C}_{s}=\mathcal{C}_{s 1} \cup \mathcal{C}_{s 2}$. Our basic assumption for this section is that whenever $C_{1}$ and $C_{2}$ are disjoint elements of $\mathcal{C}_{s 2}$, then $X \backslash\left(C_{1} \cup C_{2}\right)$ is disconnected.

In this situation, we define an equivalence relation on $\mathcal{C}_{s 2}$ by letting $C \sim C^{\prime}$ if there is a sequence of two-sided closed sets $C=C_{0}, C_{1}, \cdots, C_{n}=C^{\prime}$ with $C_{i} \cap$ $C_{i+1}=\emptyset$ for $0 \leq i \leq n-1$. In other words, each pair of adjacent $C_{i}$ 's disconnect the whole space and hence generate an irreducible partition. Let $\mathcal{E}$ be the collection of equivalence classes in $\mathcal{C}_{s 2}$ under this equivalence relation. Notice that solid sets in different equivalence classes must intersect.

Our procedure for constructing a quasi-measure on $X$ is to use a different measure for each equivalence class of two-sided closed sets. We also want to assign measures to the one-sided sets so that we obtain a solid set function. To do this, we need to classify the types of one-sided closed solid sets.

Let

$$
\begin{aligned}
& \mathcal{T}_{1}=\left\{C \in \mathcal{C}_{s 1}: \text { there is a } C^{\prime} \in \mathcal{C}_{s 2} \text { such that } C^{\prime} \subseteq C\right\}, \\
& \mathcal{T}=\left\{C \in \mathcal{C}_{s 1}: \text { for every } C \subseteq V \text { open, there is a } C^{\prime} \in \mathcal{T}_{1} \text { with } C \subseteq C^{\prime} \subseteq V\right\}, \\
& \mathcal{F}=\left\{C \in \mathcal{C}_{s 1}: \text { there is a } C^{\prime} \in \mathcal{C}_{s 1} \text { disjoint from } C \text { such that for every } D \in \mathcal{C}_{s 2}\right. \\
& \left.\quad \text { we have } C \cap D \neq \emptyset \neq C^{\prime} \cap D\right\}, \\
& \mathcal{B}=\left\{C \in \mathcal{C}_{s 1}: \text { there is a } C^{\prime} \in \mathcal{C}_{s 2} \text { such that } C^{\prime} \cap C=\emptyset\right\} .
\end{aligned}
$$

Lemma 27. Suppose that $C_{1}, C_{2} \in \mathcal{C}_{s 2}, C, C^{\prime} \in \mathcal{C}_{s}$ with $C_{1} \subseteq C, C_{2} \subseteq C^{\prime}$ and $C \cap C^{\prime}=\emptyset$. Then $C$ and $C^{\prime}$ are two-sided. Hence $C \sim C^{\prime}$.

Proof. We need only show that $X \backslash\left(C \cup C^{\prime}\right)$ is disconnected since then $\left\{C, C^{\prime}\right\}$ generates an irreducible partition of $X$. Since $C_{1}$ and $C_{2}$ are disjoint two-sided sets, our assumption on $X$ shows that $X \backslash\left(C_{1} \cup C_{2}\right)$ is disconnected. Since $o(X)=$ $g(X)=1$, there are open solid sets $U_{1}$ and $U_{2}$ with $X \backslash\left(C_{1} \cup C_{2}\right)=U_{1} \cup U_{2}$. Now $X \backslash\left(C \cup C^{\prime}\right) \subseteq U_{1} \cup U_{2}$, so if $X \backslash\left(C \cup C^{\prime}\right)$ is connected, we may assume it is contained in, say $U_{1}$. But then $X \backslash U_{1} \subseteq C \cup C^{\prime}$. Since $C_{1} \subseteq C \cap\left(X \backslash U_{1}\right)$ and $C_{2} \subseteq C^{\prime} \cap\left(X \backslash U_{1}\right)$, we see that $X \backslash U_{1}$ is disconnected, contradicting the solidity of $U_{1}$.

Lemma 28. We have $\mathcal{C}_{s 1}=\mathcal{T} \cup \mathcal{F} \cup \mathcal{B}$, a disjoint union. Hence $\mathcal{C}_{s}=\mathcal{T} \cup \mathcal{F} \cup \mathcal{B} \cup$ $\bigcup\{S: S \in \mathcal{E}\}$ is a disjoint union.

Proof. Assume that $C \in \mathcal{C}_{s 1} \backslash(\mathcal{B} \cup \mathcal{F})$. Then $C \cap C^{\prime} \neq \emptyset$ for every $C^{\prime} \in \mathcal{C}_{s 2}$. Suppose that $C \subseteq V$ where we may assume that $V$ is solid. Let $C^{\prime}=X \backslash V$. Since $C \notin \mathcal{F}$, there is a $D \in \mathcal{C}_{s 2}$ with $C^{\prime} \cap D=\emptyset$, that is $D \subseteq V$. Now, $C \cap D \neq \emptyset$, so $C \cup D$ is 
connected. Let $D^{\prime}$ be the solid hull of $C \cup D$ in $V$ (see [2 for details). Then $D^{\prime}$ is a closed, solid set contained in $V$ and containing $C \cup D$. We claim that $D^{\prime} \in \mathcal{C}_{s 1}$ which shows that $D^{\prime} \in \mathcal{T}_{1}$. This will show that $C \in \mathcal{T}$. But if $D^{\prime} \in \mathcal{C}_{s 2}$, there is a disjoint element $D^{\prime \prime} \in \mathcal{C}_{s 2}$ by proposition 11 Then $D^{\prime \prime} \cap C=\emptyset$, which shows that $C \in \mathcal{B}$, a contradiction.

Clearly, $\mathcal{F}$ and $\mathcal{B}$ are disjoint. Suppose that $C \in \mathcal{F}$. Then there is a $C^{\prime} \in \mathcal{C}_{s 1}$ which intersects every two-sided closed set and is disjoint from $C$. Then $V=X \backslash C^{\prime}$ contains $C$, but no two-sided set. Hence $C \notin \mathcal{T}$. Thus $\mathcal{F}$ and $\mathcal{T}$ are disjoint. Now suppose that $C \in \mathcal{B} \cap \mathcal{T}$. There is a two-sided closed set $D$ with $C \cap D=\emptyset$. Pick $C^{\prime} \in \mathcal{T}$ with $C \subseteq C^{\prime} \subseteq X \backslash D$. Let $D^{\prime} \subseteq C^{\prime}$ be two-sided. The preceding lemma shows that $C^{\prime} \in \mathcal{C}_{s 2}$, which contradicts that $C^{\prime} \in \mathcal{T}$.

As an example, the subset $\{0\} \times S^{1}$ of the annulus $[0,1] \times S^{1}$ is an element of $\mathcal{F}$. On the other hand, it may be shown that if $X$ is the torus, $\mathcal{F}=\emptyset$. If $C$ is the closed subset of the annulus that consists of the outside circle and an infinite spiral from the inside to the outside, then $C$ is in $\mathcal{T}$ but not the related set $\mathcal{T}_{1}$.

Lemma 29. Suppose that $C, C^{\prime} \in \mathcal{C}_{s}$ and $C \subseteq C^{\prime}$.

a) If $C \in \mathcal{T}$, then $C^{\prime} \in \mathcal{T}$.

b) If $C \in \mathcal{F}$, then $C^{\prime} \in \mathcal{F} \cup \mathcal{T}$.

c) If $C$ is two-sided with $C \in S \in \mathcal{E}$, then $C^{\prime} \in S \cup \mathcal{T}$.

Proof. a) Suppose that $C^{\prime} \subseteq V$ where we may assume that $V$ is open and solid. Since $C \subseteq V$, there is a $C^{\prime \prime} \in \mathcal{T}_{1}$ with $C \subseteq C^{\prime \prime} \subseteq V$. We may find $D$ closed and solid with $C^{\prime} \cup C^{\prime \prime} \subseteq D \subseteq V$. Then $D \in \mathcal{T}_{1}$, showing that $C^{\prime} \in \mathcal{T}$.

b) If $C^{\prime} \in \mathcal{B} \cup \mathcal{C}_{s 2}$, then there is a $D \in \mathcal{C}_{s 2}$ with $D \cap C^{\prime}=\emptyset$. But then $C \cap D=\emptyset$, so $C \notin \mathcal{F}$.

c) If $C^{\prime}$ is one-sided, then $C^{\prime} \in \mathcal{T}_{1} \subseteq \mathcal{T}$, while if $C^{\prime}$ is two-sided, then there is a disjoint $D \in \mathcal{C}_{s 2}$, so $C^{\prime} \sim D \sim C$, so $C^{\prime} \in S$.

Lemma 30. Let $C, C^{\prime} \in \mathcal{C}_{s}$ with $C \cap C^{\prime}=\emptyset$.

a) If $C \in \mathcal{T}$, then $C^{\prime} \in \mathcal{B}$.

b) If $C \in \mathcal{F}$, then $C^{\prime} \in \mathcal{F} \cup \mathcal{B}$.

c) If $C$ is two-sided and $C \in S \in \mathcal{E}$, then $C^{\prime} \in S \cup \mathcal{B}$.

Proof. a) In the definition of $\mathcal{T}$, let $V=X \backslash C^{\prime}$. We obtain a closed solid set $D \in \mathcal{T}_{1}$ with $D \cap C^{\prime}=\emptyset$. Since $D$ contains an element of $\mathcal{C}_{s 2}$, we must have that $C^{\prime} \in \mathcal{C}_{s 2} \cup \mathcal{B}$. If $C^{\prime} \in \mathcal{C}_{s 2}$, the lemma 27 shows that $D \in \mathcal{C}_{s 2}$ also, which contradicts that $D \in \mathcal{T}_{1}$. Thus $C^{\prime} \in \mathcal{B}$.

b) By definition of $\mathcal{F}, C^{\prime} \notin \mathcal{C}_{s 2}$. But $C^{\prime} \notin \mathcal{T}$ by the previous part.

c) If $C^{\prime}$ is one-sided, then, by definition, $C^{\prime} \in \mathcal{B}$. Otherwise, $C^{\prime} \in \mathcal{C}_{s 2}$, so $C \sim C^{\prime}$. Thus $C^{\prime}$ is in the same equivalence class, $S$, as $C$.

Finally, we need a lemma that will be used to show regularity for the sets in $\mathcal{B}$.

Lemma 31. If $C \in \mathcal{B}$, there is an element $C^{\prime} \in \mathcal{T}_{1}$ with $C \cap C^{\prime}=\emptyset$.

Proof. Assume not. By definition of $\mathcal{B}$, there is a $D_{1} \in \mathcal{C}_{s 2}$ with $C \cap D_{1}=\emptyset$. If $V$ is any open, solid set with $C \subseteq V \subseteq X \backslash D_{1}$, then $D_{1} \subseteq X \backslash V$. Since $X \backslash V \notin \mathcal{T}_{1}$, we must have that $X \backslash V \in \mathcal{C}_{s 2}$. 
We now claim that $X \backslash\left(C \cup D_{1}\right)$ is disconnected. This will show that $\left\{C, D_{1}\right\}$ generates an irreducible partition, so $C \in \mathcal{C}_{s 2}$, a contradiction.

To show this, let $W=X \backslash D_{1}$. By proposition [1] there is a $D_{2} \in \mathcal{C}_{s 2}$ with $C \subseteq$ int $D_{2} \subseteq D_{2} \subseteq W$. Then $W \backslash D_{2}=X \backslash\left(D_{1} \cup D_{2}\right)$ is disconnected by our assumption on $X$. Let $W \backslash D_{2}=U_{1} \cup U_{2}$, where $U_{1}$ and $U_{2}$ are open solid sets. Pick $x_{1} \in U_{1}$ and $x_{2} \in U_{2}$. If $W \backslash C=X \backslash\left(C \cup D_{1}\right)$ is connected, then there is a connected closed set $K$ with $x_{1}, x_{2} \in K \subseteq W \backslash C$.

Now let $V$ be an open solid set with $C \subseteq V \subseteq \operatorname{int}\left(D_{2}\right) \cap(W \backslash K)$. By the above, $X \backslash V \in \mathcal{C}_{s 2}$, so there is yet another $D_{3} \in \mathcal{C}_{s 2}$ with $C \subseteq D_{3} \subseteq V$. Then $W \backslash D_{3}=X \backslash\left(D_{1} \cup D_{3}\right)$ is disconnected with say, $W \backslash D_{3}=V_{1} \cup V_{2}$ with $V_{1}$ and $V_{2}$ open solid sets.

Since $D_{3} \subseteq D_{2}, U_{1} \cup U_{2}=W \backslash D_{2} \subseteq W \backslash D_{3}$. Also, $K \subseteq W \backslash V \subseteq W \backslash D_{3}$, so the connected set $U_{1} \cup U_{2} \cup K$ is contained in $V_{1} \cup V_{2}$. Thus, we may assume that $W \backslash D_{2}=U_{1} \cup U_{2} \subseteq V_{1}$. This shows that $V_{2} \subseteq W \cap X \backslash V_{1} \subseteq D_{2}$. But then $\bar{V}_{2} \subseteq D_{2}$, so $\bar{V}_{2} \cap D_{1}=\emptyset$. Since $\left\{D_{1}, D_{3}, V_{1}, V_{2}\right\}$ is an irreducible partition, this contradicts proposition 5 .

We now proceed to our construction of a family of quasi-measures on $X$. Pick $\nu$ a quasi-measure on $X$ and for each equivalence class $S \in \mathcal{E}$, let $\mu_{S}$ be a quasimeasure on $X$. We assume that $\nu(X)=\mu_{S}(X)=1$ for all $S \in \mathcal{E}$. In particular, any of the quasi-measures $\nu$, or $\mu_{S}$ may be chosen to be measures on $X$. For any closed, solid set $C$, we define

$$
\tau(C)= \begin{cases}1 & \text { if } C \in \mathcal{T} \\ \nu(C) & \text { if } C \in \mathcal{F} \\ \mu_{S}(C) & \text { if } C \in S \in \mathcal{E} \\ 0 & \text { if } C \in \mathcal{B}\end{cases}
$$

Theorem 32. The set function $\tau$ defined above is a solid set function. Thus it extends uniquely to a quasi-measure on $X$.

Proof. We show the properties of a solid set function listed after proposition 3

I: If $C_{1}, \cdots, C_{n}$ are disjoint closed solid sets contained in the closed solid set $C$, then $\sum_{k=1}^{n} \tau\left(C_{k}\right) \leq \tau(C)$.

Case 1: Some $C_{i} \in \mathcal{T}$.

In this case, for $j \neq i$, we must have $C_{j} \in \mathcal{B}$. Also $C \in \mathcal{T}$. Thus $\sum_{k=1}^{n} \tau\left(C_{k}\right)=$ $1=\tau(C)$.

Case 2: Some $C_{i} \in \mathcal{F}$.

In this case, for $j \neq i, C_{j} \in \mathcal{F} \cup \mathcal{B}$, so $\tau\left(C_{j}\right) \leq \nu\left(C_{j}\right)$. Also $C \in \mathcal{F} \cup \mathcal{T}$, so $\nu(C) \leq \tau(C)$. Thus $\sum_{k=1}^{n} \tau\left(C_{k}\right) \leq \sum_{k=1}^{n} \nu\left(C_{k}\right) \leq \nu(C) \leq \tau(C)$.

Case 3: Some $C_{i}$ is two-sided with $C_{i} \in S \in \mathcal{E}$.

Here, for $j \neq i, C_{j} \in S \cup \mathcal{B}$ and $C \in S \cup \mathcal{T}$. Thus $\sum_{k=1}^{n} \tau\left(C_{k}\right) \leq \sum_{k=1}^{n} \mu_{S}\left(C_{k}\right) \leq$ $\mu_{S}(C) \leq \tau(C)$

Case 4: All $C_{k} \in \mathcal{B}$. 
Then $\sum_{k=1}^{n} \tau\left(C_{k}\right)=0 \leq \tau(C)$.

II: If $U$ is open and solid, define $\tau(U)=1-\tau(X \backslash U)$. Then $\tau(U)=\sup \{\tau(C)$ : $\left.C \in \mathcal{C}_{s}, C \subseteq U\right\}$

Case 1: $X \backslash U \in \mathcal{T}$.

Then, if $C \subseteq U$, we have $C \in \mathcal{B}$, so $\tau(C)=0=\tau(U)$.

Case 2: $X \backslash U \in \mathcal{F}$.

First notice that $\tau(U)=1-\tau(X \backslash U)=1-\nu(X \backslash U)=\nu(U)$. If $C \subseteq U$, then $C \in \mathcal{F} \cup \mathcal{B}$, so $\tau(C) \leq \nu(C) \leq \nu(U)$. Thus $\tau(U) \geq \sup \left\{\tau(C): C \in \mathcal{C}_{s}, C \subseteq U\right\}$.

Let $\varepsilon>0$. Use regularity of $\nu$ to find a closed set $K \subseteq U$ with $\nu(K)>\nu(U)-\varepsilon$. By the definition of $\mathcal{F}$, there is a $C^{\prime} \in \mathcal{F}$ with $C^{\prime} \subseteq U$. Now use proposition 2 to find a closed solid set $C \subseteq U$ with $K \cup C^{\prime} \subseteq C$. Then $C \notin \mathcal{C}_{s 2}$ since it does not intersect $X \backslash U \in \mathcal{F}$. Since $C^{\prime} \in \mathcal{F}, C \in \mathcal{F} \cup \mathcal{T}$, so $\tau(C) \geq \nu(C) \geq \nu(K) \geq \nu(U)-\varepsilon \geq \tau(U)-\varepsilon$.

Case 3: $X \backslash U$ is two-sided and $X \backslash U \in S \in \mathcal{E}$.

If $C \subseteq U$, then $C \in S \cup \mathcal{B}$, so $\tau(C) \leq \mu_{S}(C) \leq \mu_{S}(U)=\tau(U)$.

On the other hand, if $\varepsilon>0$, we may find a closed set $K \subseteq U$ with $\mu_{S}(K)>$ $\mu_{S}(U)-\varepsilon$. Now use proposition 11 to find a two-sided set $C$ with $K \subseteq C \subseteq U$. Since $C$ is disjoint from $X \backslash U, C \sim X \backslash U$, so $C \in S$. Thus $\tau(C)=\mu_{S}(C) \geq$ $\mu_{S}(K)>\mu_{S}(U)-\varepsilon=\tau(U)-\varepsilon$.

Case 4: $X \backslash U \in \mathcal{B}$. By lemma 31, there is a $C \in \mathcal{T}_{1}$ disjoint from $X \backslash U$. Then $C \subseteq U$ and $\tau(C)=1=\tau(U)$

III. If $\left\{C_{i}\right\}_{1}^{n} \cup\left\{U_{\alpha}\right\}_{\alpha \in A}$ is an irreducible partition of $X$, then

$$
\sum \tau\left(C_{i}\right)+\sum \tau\left(U_{\alpha}\right)=1
$$

If the partition is trivial, $\mathcal{P}=\{C, X \backslash C\}$, then this is the definition of $\tau(X \backslash C)$.

On the other hand, all non-trivial partitions are of the form $\mathcal{P}=\left\{C_{1}, C_{2}, U_{1}, U_{2}\right\}$ where $C_{1}$ and $C_{2}$ are closed and $U_{1}$ and $U_{2}$ are open. Furthermore, $C_{1} \sim C_{2} \sim$ $X \backslash U_{1} \sim X \backslash U_{2}$. If we let $S \in \mathcal{E}$ be the equivalence class of these sets, then $\tau\left(C_{1}\right)+\tau\left(C_{2}\right)+\tau\left(U_{1}\right)+\tau\left(U_{2}\right)=\mu_{S}\left(C_{1}\right)+\mu_{S}\left(C_{2}\right)+\mu_{S}\left(U_{1}\right)+\mu_{S}\left(U_{2}\right)=\mu_{S}(X)=1$. This finishes the proof of the theorem.

As a corollary to this theorem, we obtain a useful construction procedure for Witt spaces with $g(X)=1$. We assume cohomology has coefficients in a field.

Corollary 33. Let $X$ be a Witt space with $g(X)=1$. For each one dimensional isotropic subspace $L$ of $H^{1}(X)$, let $\mu_{L}$ be any quasi-measure on $X$. Define $\nu$ for two-sided closed, solid sets by $\nu(C)=\mu_{L}(C)$ if the isotropic subspace associated with $C$ is $L$. For one-sided closed, solid sets, define $\nu$ as in the theorem. Then $\nu$ is a solid set function, hence extends to a quasi-measure on $X$.

Proof. Simply notice that two-sided sets with distinct isotropic subspaces are in distinct equivalence classes in the sense of the theorem.

We now give some examples that are of some interest.

First, let $X$ be the annulus, $X=S^{1} \times[0,1]$. There is only one class of two-sided closed sets. In fact, these are the solid sets that "go across" the annulus without "going around". The elements of $\mathcal{F}$, on the other hand "go around", but not "across". The elements of $\mathcal{T}$ "go around and across" and those of $\mathcal{B}$ do "neither". Let $p, q \in X$ be given. If we give elements of $\mathcal{T}$ mass 1 , those of $\mathcal{B}$ mass 0 , use the pointmass at $p$ for the two-sided sets, and use the pointmass at $q$ for the sets in $\mathcal{F}$, 
then we get a quasi-measure on $X$. It is easy to show that this quasi-measure fails to be subadditive and hence is not a measure. Notice that any contractible space can be used in place of $[0,1]$ in this example. As a further example on the annulus, we can use Lebesgue measure in the plane to measure both elements of $\mathcal{C}_{s 2}$ and $\mathcal{F}$. This will lead to a rotationally invariant quasi-measure that is not a measure.

Next, let $X=\left(S^{1}\right)^{p}$, the $p$-dimensional torus. Then $H^{1}(X)=R^{p}$, where $R$ is the coefficient ring. Let $R$ be the ring of integers. The maximal one dimensional subspaces are of the form $\left\{k\left(n_{1}, \cdots, n_{p}\right): k \in R\right\}$ where $n_{1}, \cdots, n_{p}$ are relatively prime. Hence, we get to choose a different quasi-measure for each $p$-tuple of relatively prime integers. If all these quasi-measures are chosen to be the Haar measure on $X$, the result is a "Haar" quasi-measure in the sense that it is translationally invariant in the group $X$. Alternatively, we can choose a different pointmass for each $p$-tuple as above and obtain a $\{0,1\}$-valued quasi-measure on $X$. This generalizes the case of $p=2$ given in 4 .

It should be pointed out that all known quasi-measures on, say $X=S^{1} \times[0,1]^{2}$, are of three types: the image of a quasi-measure on a space with $g=0$, an Aarnes quasi-measure such as those constructed before theorem 14, or a quasi-measure such as those in theorem 32 For spaces with larger values of $g(X)$, the only known examples are images or Aarnes quasi-measures. This is in marked contrast with the case when $g(X)=0$, where a dense collection of $\{0,1\}$-valued quasi-measures is known. It is hoped that this paper will stimulate the further developement of examples for spaces where $g(X) \geq 1$.

\section{REFERENCES}

[1] Aarnes, Johan, Quasi-states and Quasi-measures, Advances in Mathematics 86 (1991) 41-67. MR 92d:46152

[2] Aarnes, Johan, Construction of Non Subadditive Measures and Discretization of Borel Measures, Fundamenta Mathematicae 147 (1995) 213-237. [MR 96k:28022]

[3] Jacobson, Nathan, Basic Algebra I (W. H. Freeman and Company, 1985). MR 86d:00001

[4] Knudsen, Finn, Topology and the Construction of Extreme Quasi-measures, Advances in Mathematics 120 (1996), 302-321. MR 97e:28007]

Department of Mathematical Sciences, Northern Illinois University, DeKalb, IlliNOIS 60115

E-mail address: grubb@math.niu.edu 